\title{
Initiating and monitoring the evolution of single electrons within atom-defined structures
}

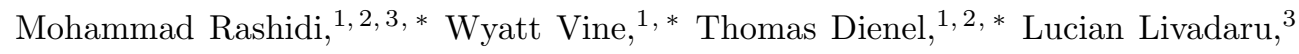 \\ Jacob Retallick, ${ }^{4}$ Taleana Huff, ${ }^{1,3}$ Konrad Walus, ${ }^{4}$ and Robert A. Wolkow ${ }^{1,2,3}$ \\ ${ }^{1}$ Department of Physics, University of Alberta, Edmonton, Alberta, T6G 2J1, Canada. \\ ${ }^{2}$ Nanotechnology Initiative, Edmonton, AB, Canada, T6G $2 M 9$ \\ ${ }^{3}$ Quantum Silicon, Edmonton, AB, Canada, T6G 2M9 \\ ${ }^{4}$ Department of Electrical and Computer Engineering, \\ University of British Columbia, Vancouver, BC, V6T 1Z4, Canada.
}

\begin{abstract}
Using a non-contact atomic force microscope we track and manipulate the position of single electrons confined to atomic structures engineered from silicon dangling bonds (DBs) on the hydrogen terminated silicon surface. By varying the probe-sample separation we mechanically manipulate the equilibrium position of individual surface silicon atoms and use this to directly switch the charge state of individual DBs. Because this mechanism is based on short range interactions and can be performed without applied bias voltage, we maintain both site-specific selectivity and single-electron control. We extract the short range forces involved with this mechanism by subtracting the long range forces acquired on a dimer vacancy site. As a result of relaxation of the silicon lattice to accommodate negatively charged DBs we observe charge configurations of DB structures that remain stable for many seconds at $4.5 \mathrm{~K}$. Subsequently we use charge manipulation to directly prepare the ground state and metastable charge configurations of DB structures composed of up to six atoms.
\end{abstract}

Atomic manipulation [1, 2] has emerged as a powerful strategy to fabricate novel atomic physical-systems 3 [ 5 . and devices [6] 9]. An important addition to this experimental toolkit would be the ability to design and control functional atomic charge configurations with single electron precision. To this end, several studies have demonstrated the ability to create, move, and controllably switch single charged species on a surface with scanning probe techniques [10 20]. One commonality of prior charge manipulation studies is that they have relied upon the application of bias voltage to induce charge transitions. In most cases this results in a non-negligible tunneling current, whereas in principle charge manipulation could be performed by transferring single electrons. Two recent works highlight progress in this area: Steurer et al. [16] have demonstrated the lateral manipulation of charge between pentacene molecules adsorbed to a $\mathrm{NaCl}$ thin film and Fatayer et al. 20] have performed charge manipulation with zA tunneling currents.

Building on these efforts we present the manipulation of charge within engineered atomic nanostructures based on single electron events at zero applied bias voltage $(0 \mathrm{~V})$. We investigate atom defined charge configurations composed of patterned silicon DBs on a hydrogenterminated $\operatorname{Si}(100)-2 \times 1$ surface. One advantage to working on the silicon surface is that because DBs are midgap states they are electronically isolated from the bulk substrate [21]. They can therefore localize charge without the requirement of a thin insulating film between structure and substrate, which has been essential in many previous studies [10 16, 20, 22, 23. The regular spacing of DBs is also guaranteed by the crystal lattice. Recent advances in patterning of DBs now allows large errorfree structures to be created $[24-26]$. Recent non-contact atomic force microscopy (nc-AFM) measurements 27. have confirmed that the energy of the negative to neutral charge transition of an isolated DB on a highly ndoped sample is close to the bulk Fermi level (within a few hundred $\mathrm{meV}$ ). This enables the charge state of DBs to be selectively modified by shifting this charge transition level above or below the bulk Fermi level with bias voltage or other nearby charged DBs $9,21,27,28$. In contrast, here we demonstrate that the charge manipulation of DBs can also be achieved mechanically by using the probe to directly manipulate the equilibrium position of the host atom, making it favorable to host a negative charge. Because this ability is based on short range interactions between the probe and the target atom and can be performed at $0 \mathrm{~V}$ we maintain both site-specific selectively and single-electron control.

All experiments were performed on an Omicron LT STM/AFM operating at $4.5 \mathrm{~K}$ and ultrahigh vacuum $\left(<1 \times 10^{-10}\right.$ Torr $)$. Tips were created from polycrystalline tungsten wire that was chemically etched, sharpened with a focussed ion beam, and attached to a qPlus sensor [29]. The tips had resonance frequencies of $28 \mathrm{kHz}$, Q-factors between $12 \mathrm{k}$ and $14 \mathrm{k}$, and were driven with an amplitude of $50 \mathrm{pm}$. Frequency shift measurements were converted to force using the Sader-Jarvis method [30, 31. A stiffness of $1800 \mathrm{~N} / \mathrm{m}$ for the frequency shift to force conversion was assumed. An additional electrode on the sensor was used to supply tunneling current. Tips were further sharpened by nitrogen etching while performing field ion microscopy [32. In-situ tip processing was performed by controlled contacts of the tip to the sample surface which likely results in a decoration of the tip apex with silicon atoms 25, 33, 34. Samples were cleaved from highly arsenic doped $\left(1.5 \times 10^{19}\right.$ 

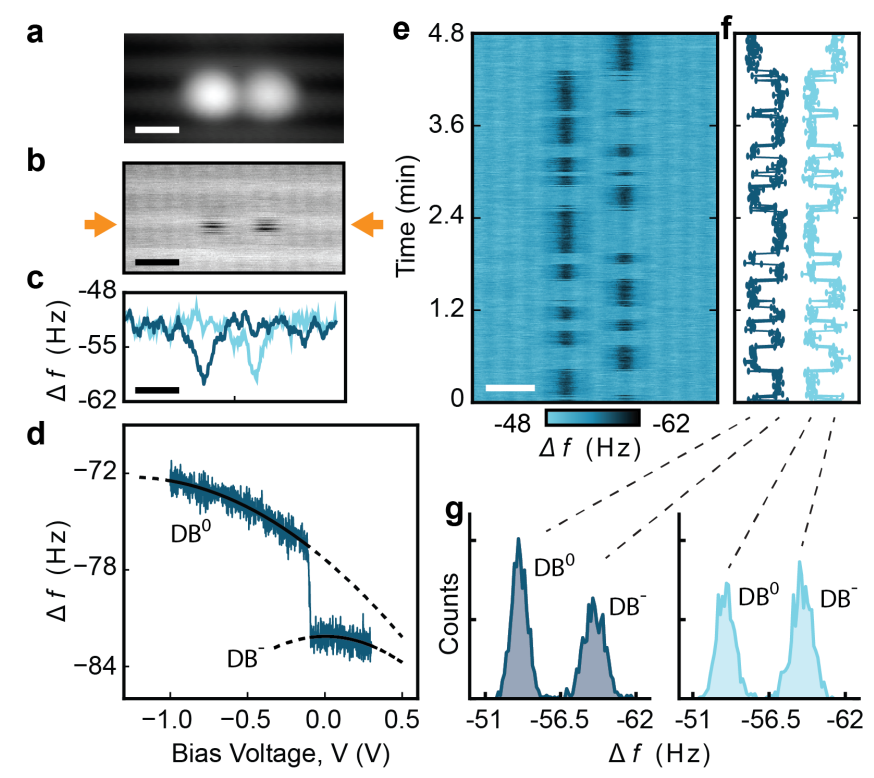

FIG. 1. Charge configurations of two closely-spaced DBs. (a) Constant current filled state STM image, $-1.8 \mathrm{~V}, 50 \mathrm{pA}$. (b) Constant height $\Delta f$ image, $0 \mathrm{~V}, 300 \mathrm{pm}$. (c) Two constant height $\Delta f$ line scans $(0 \mathrm{~V},-300 \mathrm{pm})$ at the position indicated by the orange arrows in (b). (d) $\Delta f(V)$ spectroscopy taken above an isolated DB $(-370 \mathrm{pm})$. The two individual segments have been fitted by two parabolas (solid lines: fit, dashed lines: extrapolation) corresponding to the neutral and negatively charged states $\left(\mathrm{DB}^{0}\right.$ and $\mathrm{DB}^{-}$, respectively). (e) Combined map of 400 constant height $\Delta f$ line scans $(0 \mathrm{~V}$, $-300 \mathrm{pm}$ ) taken sequentially over a 4.8 minute period. (f) Time-dependent bistable signal for the two individual DBs extracted from (e). (g) Histograms of the signals in (e). Labels indicate the charge state assignment of each peak. Scale bar is $1 \mathrm{~nm}(\mathrm{a}-\mathrm{c}, \mathrm{e})$.

atom $/ \mathrm{cm}^{3}$ ) (100)-oriented Si crystals. After degassing at $600{ }^{\circ} \mathrm{C}$ for 12 hours, samples were flash annealed to temperatures as high as $1250{ }^{\circ} \mathrm{C}$ before passivating the surface with hydrogen while maintaining a sample temperature of $330{ }^{\circ} \mathrm{C}$. The high flash temperatures have been previously shown to induce a dopant depletion region extending as far as $100 \mathrm{~nm}$ below the sample surface [35, 36. DBs were patterned by applying short voltage pulses $(+2.1 \mathrm{~V}, 10 \mathrm{~ms})$ with the tip positioned directly above hydrogen [37. All tip offsets $(\Delta z)$ used within the manuscript are in reference to an STM setpoint of $-1.8 \mathrm{~V}$ and $50 \mathrm{pA}$ measured over hydrogen.

In Figure 1, two DBs are patterned with two intervening hydrogen atoms using voltage pulses applied to the probe (Fig. 1a). Pairs of DBs are known to host only a single negative charge because the Coulombic repulsion between two closely-spaced negative charges would otherwise be too large 21]; here, constant height frequency shift $(\Delta f)$ images of the pair appear streaky because the negative charge switches sites multiple times over the time it takes to acquire an image (Fig. 1b). This is seen clearly in individual $\Delta f$ line scans across the structure (Fig. 1c, taken at the position of the orange arrows in Fig. 1b) which reveal the localization of charge to one $\mathrm{DB}$, with subsequent line scans demonstrating that this charge occasionally switches to the other DB. To definitively assign the change in contrast observed over each DB in $\Delta f$ images to a charge state, we performed biasdependent $\Delta f$ spectroscopy $(\Delta f(V))$ on an isolated $\mathrm{DB}$ (Fig. 1d) which is negatively charged at $0 \mathrm{~V}$ on highly n-doped samples [27] (also see Supporting Information, Height-dependent contrast in $\Delta f$ images). It reveals a sharp transition between two parabolas [12, associated with switching between the neutral (left of the step) and negatively charged states of the DB. By comparing the $\Delta f$ of the negatively charged state measured at $0 \mathrm{~V}$ to the fit of the neutral state's parabola at $0 \mathrm{~V}$ it becomes clear that the dark contrast (larger $|\Delta f|$ ) in Figure 1b,c corresponds to the negatively charged DB.

By stacking sequential $\Delta f$ line scans (Fig. 1f), we can monitor the charge switching between the two sites in real-time. Previous theoretical estimates for the tunneling rate between two closely-spaced DBs have ranged from $\mathrm{THz}$ to $\mathrm{GHz}$, depending on the spacing [38, 39. Surprisingly, the bistable signal for each DB extracted from Fig. 1f demonstrates that the system's charge configuration often remains stable for seconds (Fig. 1g). Recent studies have revealed that charged species are often stabilized by a lattice relaxation of the supporting substrate [10, 20, 40]. Density functional theory has similarly shown that negatively charged silicon DBs experience approximately $200 \mathrm{meV}$ stabilization due to a relaxation of the lattice, which results in the nuclear position of the host atom being raised by approximately $30 \mathrm{pm}$ relative to the neutral state [1143]. In this case, the lattice relaxation prevents the electron from elastically tunneling between the paired DBs. To assign the position of the charge in each $\Delta f$ line scan each trace was fitted with two Gaussian profiles. Histograms of the determined $\Delta f$ center values demonstrate two Gaussian profiles, representing the negative and neutral charge states of each DB (Fig. 1h and Supporting Information Fig. S1). Because they are well separated, the charge state of each DB can be assigned reliably by a single line scan (Supporting Information Fig. S3).

Interestingly, we found that the occupation of $\mathrm{DB}$ structures observed at $0 \mathrm{~V}$ appeared to depend strongly on $\Delta z$. In Figure 2 we demonstrate this by performing a series of constant height line scan maps on a structure composed of six DBs with different $\Delta z$. The average occupation of each DB at each height can be inferred from the histograms of the $\Delta f$ measured over each $\mathrm{DB}$ (Supporting Information, Fig. S3). More simply, the average occupation of the entire structure can be inferred by counting the number of dark bars in each line scan map. At the tip's closest approach $(-320 \mathrm{pm}$, top panel Fig. 2b) all six DBs appear negatively charged. Upon 

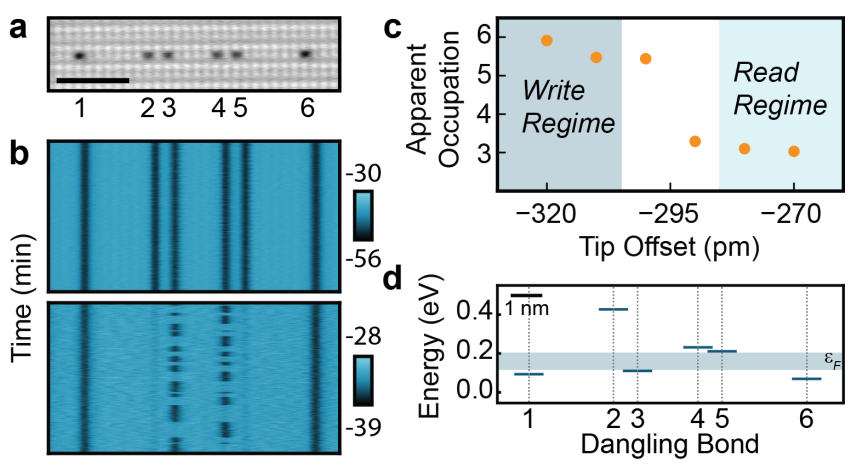

FIG. 2. Evolution of charge configurations of a symmetric six DB structure at different tip heights. (a) Constant height $\Delta f$ image $(0 \mathrm{~V},-300 \mathrm{pm})$. (b) Maps of 800 constant height $\Delta f$ line scans acquired over 18 minutes at $-320 \mathrm{pm}$ (top panel) and $-270 \mathrm{pm}$ (bottom panel). The scale bar in (a) is $3 \mathrm{~nm}$ and applies to (b). Color bars correspond to $\Delta f$. Histograms of the $\Delta f$ extracted over each DB in (b) are available in Supplementary Information Fig. S3. (c) The average occupation of the structure inferred from digitizing the charge configuration at different $\Delta z$. Two interaction regimes: read and write are indicated. (d) Energetic shift of the neutral to negative charge transition (0/-) levels of each DB in the structure $(1,3$, and 6 are negatively charged) with respect to the (0/-) level of an isolated DB $(0 \mathrm{eV})$. The levels are shifted Coulombically by the negative charges confined to the structure and are calculated in the absence of the probe using an electrostatic approximation of point charges and a surface dielectric constant of 6.35 . Because the exact energy of the (0/-) level is unknown we indicate a range of energies (blue shaded area) over which the bulk Fermi level would give rise to the charge configurations observed in the lower panel of (b).

withdrawing the tip by just $50 \mathrm{pm}(-270 \mathrm{pm}$, Fig. 2b bottom panel) only three DBs are negatively charged at any given moment. This change in the apparent timeaveraged occupation of the structure does not vary linearly with $\Delta z$, but instead transitions sharply between -300 and -290 pm (Fig. 2c).

To understand this trend we performed distancedependent $\Delta f$ spectroscopy $(\Delta f(z))$ at $0 \mathrm{~V}$ on the individual DBs in a pair (Fig. 3a, blue curves) and over a vacancy on the surface (Fig. 3a, orange curve). We begin by withdrawing the tip $700 \mathrm{pm}$ from our reference height to effectively eliminate the forces between the tip and sample and subsequently walk the tip towards the sample to progressively reintroduce them. Until approximately $\Delta z=-100 \mathrm{pm}$ all three curves are nearly identical, confirming that the long range forces (i.e. van der Waals and capacitive forces due to the contact potential difference) are dominant [44 46]. Focusing on the approach curve obtained over the DB we note that at $\Delta z=-302 \pm 2 \mathrm{pm}$ there is a sudden increase in the $|\Delta f|$ (observed at $\Delta z=-301 \pm 2 \mathrm{pm}$ on the other $\mathrm{DB}$ ). Crucially, we note that this results in hysteresis between the approach and retract curves, with the $|\Delta f|$ measured in the latter remaining larger until approximately
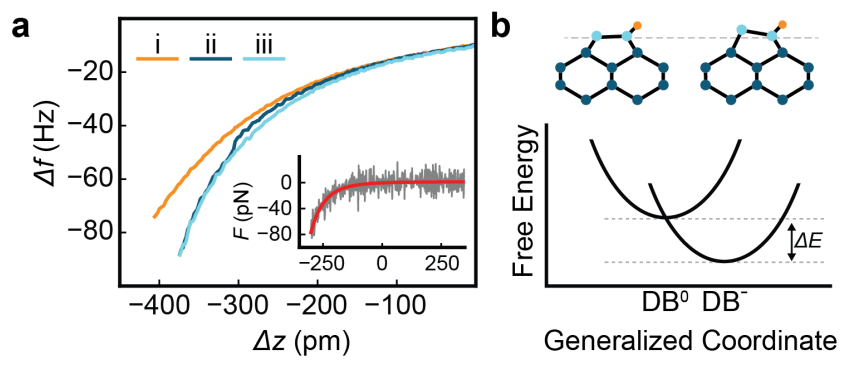

FIG. 3. Mechanically induced charge switching of a DB in a pair. (a) $\Delta f(z)$ at $0 \mathrm{~V}$ taken above an $\mathrm{H}$ atom $(i)$ and on a DB in a pair ( $i i$ - approach; iii - retract). Inset: the short range forces acting on the neutral DB (curve ii in (a)) up to the sharp step observed in $|\Delta f|$. See SI for more details. (b) Top panel: sketch of the equilibrium position of the host atom of a neutral DB (left) and a negatively charged DB (right). Dark blue, light blue, and orange atoms represent bulk silicon, silicon dimers, and hydrogen, respectively. Bottom panel: Free energy diagram depicting the neutral to negative charge transition for a DB due to the mechanical displacement of the host atom by the tip. $\Delta E$ corresponds to the lattice relaxation energy.

$\Delta z=-100 \mathrm{pm}$. Because of the similarity between the step in the approach curve and those observed in $\Delta f(V)$ experiments (e.g. Fig. 1d) we attribute this phenomenon to the localization of the pair's charge to the DB beneath the tip. Two observations confirm this: if a step is observed in the $\Delta f(z)$ obtained over one $\mathrm{DB}$, subsequent $\Delta f(z)$ curves taken over the same DB do not demonstrate this behaviour. Instead, both the approach and retract curves trace the curve with the greater $|\Delta f|$, indicating the DB remains charged. On the other hand, if a step is observed in the $\Delta f(z)$ obtained over one $\mathrm{DB}$ and the subsequent $\Delta f(z)$ is performed on the other the hysteresis is consistently observed, indicating we caused the charge to switch sites.

Similar hysteresis in $\Delta f(z)$ curves have been observed on the bare $\mathrm{Si}(100)$ surface previously [47. In their case, the presence of sudden hysteretic steps corresponded directly to a toggling of the buckling direction of a single $\mathrm{Si}(100)$ dimer on the hydrogen-free surface. The authors concluded that at small absolute tip heights short range forces between the probe and sample result in a mechanically-induced deformation of the lattice. The same mechanism is at play in our experiments. One distinction of our work is that the mechanical deformation also corresponds to a change in the charge state of the surface atom. This can be understood by considering the equilibrium positions of the host silicon atom for a negative and a neutral DB, which as noted earlier, differ due to relaxation of the lattice (sketch in top panel, Fig. 3b) 4143]. Because the forces are all attractive at the height corresponding to the step in the approach curve, a displacement of the surface atom is expected to be in the direction of the tip, causing the atom to 


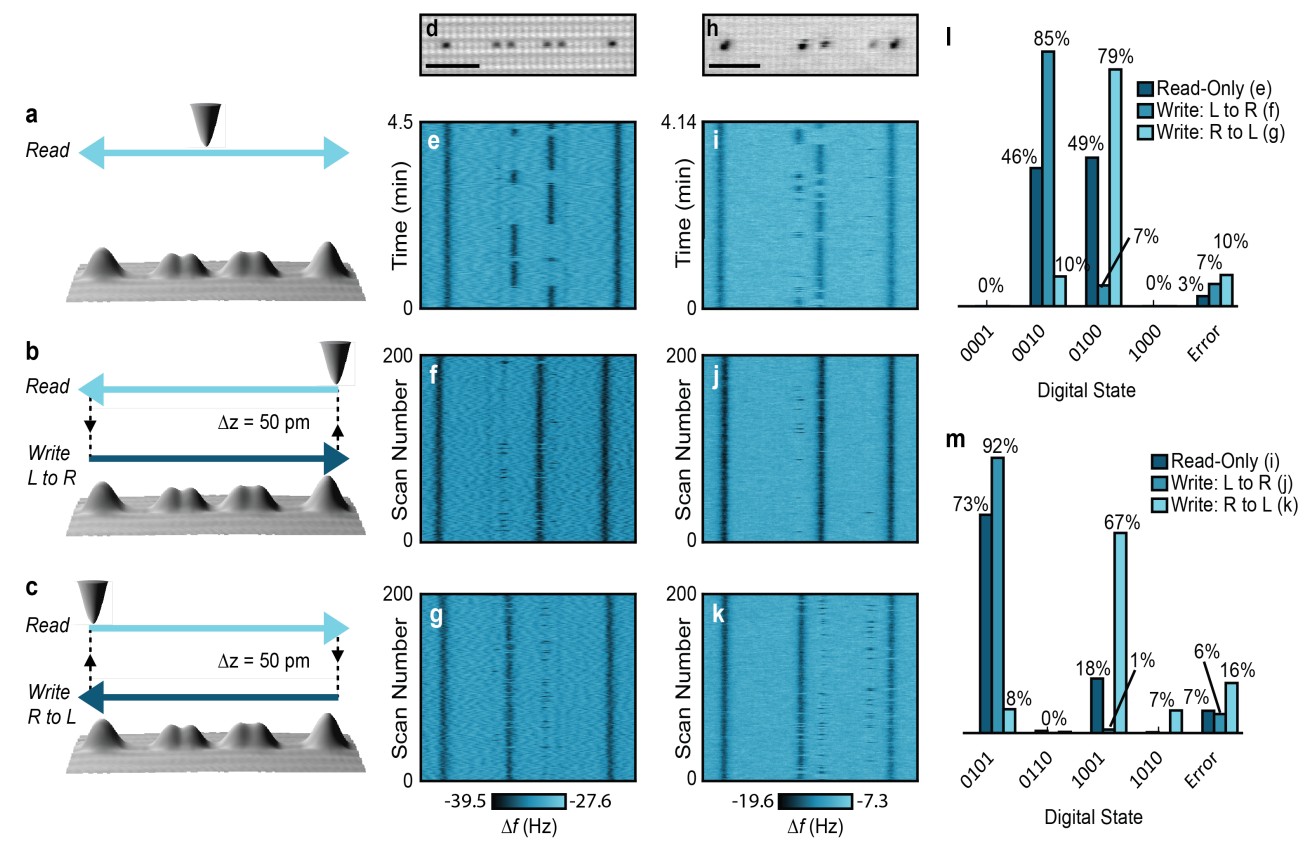

FIG. 4. Controlled preparation of charge configurations in symmetric and asymmetric DB structures. Visualization of the scan modes: (a) all measurements are restricted to the read regime; (b) The tip is scanned from left to right $(L$ to $R$ ) in the write regime, retracted $50 \mathrm{pm}$ to the read regime, and scanned back across; (c) the same process as (b) with directions reversed (write $R$ to $L)$. (d) Constant height $\Delta f$ image of the symmetric six-DB structure $(0 \mathrm{~V},-300 \mathrm{pm})$. (e-g) Maps of 200 line scans across structure in (d) corresponding to scheme (a) shown in (e), scheme (b) shown in (f), and scheme (c) shown in (g) (write regime: $-320 \mathrm{pm}$, read regime: $-270 \mathrm{pm})$. (h) Constant height $\Delta f$ image of the asymmetric five-DB structure, $-350 \mathrm{pm}, 0 \mathrm{~V}$. (i-k) Maps of 200 line scans across structure (i) corresponding to scheme (a) (i), scheme (b) (j), and scheme (c) (k) (write regime: $-370 \mathrm{pm}$, read regime: $-320 \mathrm{pm}$ ). (1) Histograms of the binary numbers determined from digitization of the line scans in (e-g). 0's and 1's correspond to neutral and negatively charged DBs, respectively. Only the four interior DBs are considered. (m) Histograms of the binary numbers determined from digitization of the line scans in (i-k). The scale bars in (d) and (i) are $3 \mathrm{~nm}$.

re-hybridize and adopt greater $s p^{3}$ character. Consequently, the total free energy of the negatively charged state is lowered with respect to the neutral state, leading to the charging of the DB beneath the tip (bottom panel, Fig. 3b). Using $\Delta f(z)$ curves obtained over dimer vacancies on the surface (Supporting Information Fig. S4) we separate the long and short range force contributions $44-$ 46]. We find the short range forces required to lift the equilibrium position of the neutral host atom is fit best by a function of the form $-\mathrm{C} / z^{7}$, where $z$ is the absolute tip height and $\mathrm{C}$ is a constant (Supporting Information Fig. S6). This strongly suggests that van der Waals forces are responsible for displacing the host atom [48. A force of $-75 \pm 13 \mathrm{pN}(-77 \pm 12 \mathrm{pN})$ was found for the right (left) DB (inset Fig. 3a, uncertainty in force measurement corresponds to one standard deviation) which is consistent with the force reported by Sweetman et. al. required to toggle the Si dimer [34, 47].

The experiments in Figure 2 can now be clearly explained. At small absolute tip heights the short range forces are strong enough that as the probe scans over the structure the charging of each DB becomes favorable whenever it is beneath the tip (top panel, Fig. $2 \mathrm{~b}$ ).
This necessitates that electrons vacate prior negatively charged DBs such that the overall occupation of the structure remains constant. Upon withdrawing the tip a short distance, however, this effect is greatly diminished. As a result we observe that specific charge configurations can remain stable for many sequential measurements ( $>15 \mathrm{~s}$ on average, bottom panel, Fig. $2 \mathrm{~b}$ ). It is also interesting to note that only two charge configurations appear consistently: the two outer DBs remain continuously charged and a single negative charge is observed to switch between the two central DBs, similar to the behavior observed on an isolated pair. By observing that the total amount of time the central charge spends in the left DB (50\%) is roughly equal to the right (46\%), and noting the structure's symmetry, it is clear that these two charge configurations correspond to the degenerate ground state. We do not observe higher energy charge configurations of this structure, likely because the Coulombic interaction between closely spaced negative charges makes them energetically unfavorable, e.g., if $\mathrm{DBs} 1,2$, and 6 in Fig. 2d were negatively charged. We therefore identify two interaction regimes (Fig. 2c): one where charge can be controllably manipulated by 
the tip (the write regime) and another where we can observe stable or metastable charge configurations (the read regime).

To further validate our assignment of the write and read regimes we performed the experiments depicted in schemes Figures $4 \mathrm{a}-\mathrm{c}$ on the symmetric structure (Fig. 4d) and an asymmetric structure composed of five DBs (Fig. 4h). First, we restricted the measurements to the read regime (scheme Fig. 4a, Fig. 4e,i), allowing us to characterize the intrinsic charge configurations of the structures and assess their relative energies based on how often they occur (dark blue in Fig. 4l,m). Subsequent experiments contain two associated phases: in the write phase, the tip is scanned across the structure at close proximity; in the read phase, the tip is retracted $50 \mathrm{pm}$ with respect to the write phase and scanned back across (depicted in schemes Fig. 4b,c). It might be expected that any charge configuration prepared by the write phase should be observed in the read phase. Indeed, Figure $4 \mathrm{f}, \mathrm{g}$ and $4 \mathrm{j}, \mathrm{k}$ confirm that charge in the interior of both structures can be manipulated. On the symmetric structure we could consistently initiate charge to the right (Fig. 4f, 85\%) or left (Fig. 4g, 79\%) central DB, corresponding to preparation of the degenerate ground state configurations observed in Figure 4e. On the asymmetric structure measurements restricted to the read regime (Fig. 4i) demonstrate that this system has three negative charges. On this structure also only the charge confined to the inner pair fluctuates, but because the structure is asymmetric these two charge configurations are non-degenerate. Although we expected the interior charge to favor the left DB of the pair we observe the opposite (Fig. 4i,m 18\% vs. $73 \%$, respectively). This indicates that other charged species (e.g. DBs or ionized donors) act as an additional electrostatic bias on this structure. We note, however, that these hidden biases can be counteracted by patterning additional DBs in the structure's surrounding area (Supporting Information, Fig. S5). Using the techniques previously described the central charge could be manipulated to selectively occupy the right (Fig. 4j, 92\%) or left (Fig. 4k. 67\%) DB of the pair, demonstrating that in addition to the ground state configurations the occurrence of metastable charge configurations can also be enhanced (Fig. 4i).

These results demonstrate that single electrons can be sensed and manipulated within structures derived from DBs. We find that charge configurations can remain stable for periods on the order of several seconds as a result of a relaxation of the silicon lattice which acts to stabilize negatively charged DBs. Using the probe, specific charge configurations can be prepared. The mechanism used to achieve this does not depend on the application of bias voltage-instead it is due to a mechanical manipulation of the DB's host atom with the probe which initiates a change of its charge state. The techniques presented here expand the scanning probe toolkit with the ability to position charge within atomic structures and prepare desired charge configurations.

We would like to thank Mark Salomons and Martin Cloutier for their technical expertise. We also thank Leo Gross and Gerhard Meyer for stimulating discussions. We thank NRC, NSERC, and AITF for their financial support.

M.R., W.V. and T.D. contributed equally to this work.

* Correspondence to: rashidi@ualberta.net, wyattvine@gmail.com, thdienel@gmail.com

[1] D. K. Schweizer and E. K. Eigler, Nature 344, 524 (1990).

[2] Y. Sugimoto, P. Pou, O. Custance, P. Jelinek, M. Abe, R. Perez, and S. Morita, Science 322, 413 (2008).

[3] M. R. Slot, T. S. Gardenier, P. H. Jacobse, G. C. P. van Miert, S. N. Kempkes, S. J. M. Zevenhuizen, C. M. Smith, D. Vanmaekelbergh, and I. Swart, Nat. Phys. 13, 672 (2017).

[4] R. Drost, T. Ojanen, A. Harju, and P. Liljeroth, Nat. Phys. 13, 668 (2017).

[5] S. Fölsch, J. Martínez-Blanco, J. Yang, K. Kanisawa, and S. C. Erwin, Nat. Nanotechnol. 9, 505 (2014).

[6] A. A. Khajetoorians, J. Wiebe, B. Chilian, and R. Wiesendanger, Science 332, 1062 (2011).

[7] M. Fuechsle, J. A. Miwa, S. Mahapatra, H. Ryu, S. Lee, O. Warschkow, L. C. L. Hollenberg, G. Klimeck, and M. Y. Simmons, Nat. Nanotechnol. 7, 242 (2012).

[8] F. E. Kalff, M. P. Rebergen, E. Fahrenfort, J. Girovsky, R. Toskovic, J. L. Lado, J. Fernández-Rossier, and A. F. Otte, Nat. Nanotechnol. 11, 926 (2016).

[9] T. Huff, H. Labidi, M. Rashidi, R. Achal, L. Livadaru, T. Dienel, J. Pitters, and R. A. Wolkow, arXiv:1706.07427 (2017)

[10] J. Repp, G. Meyer, F. E. Olsson, and M. Persson, Science 305, 493 (2004).

[11] G. V. Nazin, X. H. Qiu, and W. Ho, Phys. Rev. Lett. 95, 166103 (2005).

[12] L. Gross, F. Mohn, P. Liljeroth, J. Repp, F. J. Giessibl, and G. Meyer, Science 324, 1428 (2009).

[13] M. Sterrer, T. Risse, U. M. Pozzoni, L. Giordano, M. Heyde, H. P. Rust, G. Pacchioni, and H. J. Freund, Phys. Rev. Lett. 98, 096107 (2007).

[14] T. Leoni, O. Guillermet, H. Walch, V. Langlais, A. Scheuermann, J. Bonvoisin, and S. Gauthier, Phys. Rev. Lett. 106, 216103 (2011).

[15] W. Steurer, J. Repp, L. Gross, I. Scivetti, M. Persson, and G. Meyer, Phys. Rev. Lett. 114, 036801 (2015).

[16] W. Steurer, S. Fatayer, L. Gross, and G. Meyer, Nat. Commun. 6, 8353 (2015).

[17] S. D. Bennett, L. Cockins, Y. Miyahara, P. Grutter, and A. A. Clerk, Phys. Rev. Lett. 104, 017203 (2010).

[18] K. Teichmann, M. Wenderoth, S. Loth, J. K. Garleff, a. P. Wijnheijmer, P. M. Koenraad, and R. G. Ulbrich, Nano lett. 11, 3538 (2011).

[19] M. Setvin, J. Hulva, G. S. Parkinson, M. Schmid, and U. Diebold, Proc. Natl. Acad. Sci. , E2556 (2017).

[20] S. Fatayer, B. Schuler, W. Steurer, I. Scivetti, J. Repp, L. Gross, M. Persson, and G. Meyer, Nat. Nanotechnol. 13, 376 (2018). 
[21] M. B. Haider, J. L. Pitters, G. A. DiLabio, L. Livadaru, J. Y. Mutus, and R. A. Wolkow, Phys. Rev. Lett. 102, 046805 (2009).

[22] L. Liu, T. Dienel, R. Widmer, and O. Gröning, ACS Nano 9, 10125 (2015).

[23] F. Schulz, M. Ijäs, R. Drost, S. K. Hämäläinen, A. Harju, A. P. Seitsonen, and P. Liljeroth, Nat. Phys. 11, 229 (2015).

[24] N. Pavliček, Z. Majzik, G. Meyer, and L. Gross, Appl. Phys. Lett. 111, 053104 (2017).

[25] T. R. Huff, H. Labidi, M. Rashidi, M. Koleini, R. Achal, M. H. Salomons, and R. A. Wolkow, ACS Nano 11, 8636 (2017).

[26] R. Achal, M. Rashidi, J. F. Croshaw, D. Churchill, M. Taucer, T. Huff, M. Cloutier, J. Pitters, and R. A. Wolkow, Nat. Commun. XX, XXX (2018).

[27] M. Rashidi, E. Lloyd, T. R. Huff, R. Achal, M. Taucer, J. J. Croshaw, and R. A. Wolkow, ACS Nano 11, 11732 (2017).

[28] M. Taucer, L. Livadaru, P. G. Piva, R. Achal, H. Labidi, J. L. Pitters, and R. A. Wolkow, Phys. Rev. Lett. 112, 256801 (2014).

[29] F. J. Giessibl, Appl. Phys. Lett. 76, 1470 (2000).

[30] J. E. Sader and S. P. Jarvis, Appl. Phys. Lett. 84, 1801 (2004).

[31] J. Welker, E. Illek, and F. J. Giessibl, Beilstein J. Nanotechnol. 3, 238 (2012).

[32] M. Rezeq, J. Pitters, and R. Wolkow, J. Chem. Phys. 124, 204716 (2006).

[33] H. Labidi, M. Koleini, T. Huff, M. Salomons, M. Cloutier, J. Pitters, and R. A. Wolkow, Nat. Commun. 8, 14222 (2017).

[34] S. Jarvis, A. Sweetman, J. Bamidele, L. Kantorovich, and P. Moriarty, Phys. Rev. B 85, 235305 (2012).

[35] J. L. Pitters, P. G. Piva, and R. A. Wolkow, J. Vac. Sci. Technol., B 30, 021806 (2012).

[36] M. Rashidi, J. A. J. Burgess, M. Taucer, R. Achal, J. L. Pitters, S. Loth, and R. A. Wolkow, Nat. Commun. 7, 13258 (2016).

[37] J. W. Lyding, T. C. Shen, J. S. Hubacek, J. R. Tucker, and G. C. Abeln, Appl. Phys. Lett. 64, 2010 (1994).

[38] L. Livadaru, P. Xue, Z. Shaterzadeh-Yazdi, G. A. DiLabio, J. Mutus, J. L. Pitters, B. C. Sanders, and R. A. Wolkow, New J. Phys. 12, 083018 (2010).

[39] Z. Shaterzadeh-Yazdi, B. C. Sanders, and G. A. DiLabio, J. Chem. Phys. 148, 154701 (2018).

[40] F. E. Olsson, S. Paavilainen, M. Persson, J. Repp, and G. Meyer, Phys. Rev. Lett. 98, 176803 (2007).

[41] J. E. Northrup, Phys. Rev. B 40, 5875 (1989).

[42] S. R. Schofield, P. Studer, C. F. Hirjibehedin, N. J. Curson, G. Aeppli, and D. R. Bowler, Nat. Commun. 4, 1649 (2013).

[43] H. Kawai, O. Neucheva, T. Leh, C. Joachim, and M. Saeys, Surf. Sci. 645, 88 (2016).

[44] M. A. Lantz, H. J. Hug, R. Hoffmann, P. J. A. van Schendel, P. Kappenberger, S. Martin, A. Baratoff, and H.-J. Güntherodt, Science 291, 2580 (2001).

[45] M. Ternes, C. González, C. P. Lutz, P. Hapala, F. J. Giessibl, P. Jelínek, and A. J. Heinrich, Phys. Rev. Lett. 106, 016802 (2011).

[46] A. Sweetman and A. Stannard, Beilstein J. Nanotechnol. 5, 386 (2014).

[47] A. Sweetman, S. Jarvis, R. Danza, J. Bamidele, S. Gangopadhyay, G. A. Shaw, L. Kantorovich, and P. Mori- arty, Phys. Rev. Lett. 106, 136101 (2011).

[48] F. Bocquet, L. Nony, and C. Loppacher, Phys. Rev. B 83, 035411 (2011). 


\section{Supplementary Information: \\ Initiating and monitoring the evolution of single electrons within atom-defined structures

\author{
Mohammad Rashidi ${ }^{1,2,3^{*}}$, Wyatt Vine ${ }^{1^{*}}$, Thomas Dienel ${ }^{1,2^{*}}$, Lucian Livadaru ${ }^{3}$, Jacob Retallick ${ }^{4}$, \\ Taleana Huff ${ }^{1,3}$, Konrad Walus ${ }^{4}$, Robert A. Wolkow ${ }^{1,2,3}$ \\ ${ }^{1}$ Department of Physics, University of Alberta, Edmonton, AB, Canada, T6G 2R3 \\ ${ }^{2}$ Nanotechnology Initiative, Edmonton, AB, Canada, T6G 2M9 \\ ${ }^{3}$ Quantum Silicon, Edmonton, AB, Canada, T6G 2M9 \\ ${ }^{4}$ Department of Electrical and Computer Engineering, University of British Columbia, Vancouver, BC, Canada, V6T 1Z4 \\ *Authors contributed equally \\ Correspondence to: rashidi@ualberta.net,wyattvine@gmail.com,thdienel@gmail.com
}

Table of Contents:

- Definition and discussion of error rate

- Data processing for repeated line scan experiments and assignment of digital charge configurations

- Height-dependent contrast in $\Delta f$ images

- Frequency to force conversion

- Additional experimental data

- References for Supporting Information

Error Rate: Throughout measurements restricted to the read-regime we occasionally observed negative charges occupying both dangling bonds in a pair, despite this being unlikely due to Coulombic repulsion. We define these line scans as errors. While it was typically several percent we have achieved error rates of $<1 \%$ (Supporting Information, Fig. S2). We have identified several contributing factors. (i) The read and write regimes are sensitive to the tip height (Fig. 2 and SI Fig. S3). Accordingly, we find that small changes in tip height (e.g. noise of the tuning fork's amplitude) can occasionally result in unintentional manipulation of the charge state of dangling bonds beneath the tip while in the read-regime. This can result in an increase to the apparent occupation of the structure (e.g. Fig. 2). It can also reduce the success-rate of charge manipulation in the write-regime. (ii) Sharp tips were found to more clearly resolve the two charge states of each dangling bond, i.e., better signal to noise ratio. This reduces the number of incorrect charge state assignments, which are performed in a digital fashion. Similarly, with H-terminated tips, which can be effectively identified via force distance spectroscopy [1], it was more difficult to discriminate the two charge states of each dangling bond.

Data processing for repeated line scan experiments and assignment of digital charge configurations: Minimal data processing was performed, and raw data was used whenever possible. All experiments with repeated line scans were performed in constant height mode. For experiments performed entirely in the read regime, forward and backward line scans, which are saved in separate files by the control software, were aligned manually by removing an equal number of pixels at the start of both scans and zipped 
together (step 1, Fig. S1b). Measurements often exceeded 30 minutes, over which time the tip would inevitably drift towards or away from the surface due to piezo creep and thermal drift. To account for this, a linear drift was subtracted from all measurements with repeated line scans by fitting the average $\Delta f$ for each line scan over the course of an experiment (step 2, Fig. S1c). In experiments where $\Delta f$ drifted by more than $2 \mathrm{~Hz}$ the entire run was rejected.

The $\Delta f$ value measured over each dangling bond was extracted by independently fitting each dangling bond associated peak in the line scans (defined by a 30-pixel window centered on their position) with a Gaussian function (step 3, Fig. S1d). Supporting Figure S1e,f shows the extracted $\Delta f$ values for two dangling bonds. The bistable behavior of each dangling bond is clearly visible.

Binary numbers were assigned to the charge states by making a single cut in $\Delta f$ (Fig. S1e,f demonstrate cuts). Dangling bonds with $|\Delta f|$ greater than the cut were assigned a negative charge state, while those with $|\Delta f|$ smaller than the cut were assigned a neutral charge state (Fig. S1e,f). Two additional steps were used to create the histograms in Fig. S3 and S4. First, the smallest $|\Delta f|$ in the set of the $\Delta f$ extracted for all the dangling bonds in an experiment (corresponding to a fit of the background) was set to 0 (step 4). Thus, the normalized $\Delta f$ for all the dangling bonds would be positive. Second, each $\Delta f$ was normalized by setting the average $\Delta f$ for the two isolated dangling bonds to 1.0 (step 5). Because the isolated dangling bonds were always negatively charged, a normalized $\Delta f$ of 1.0 corresponds to the average $\Delta f$ for a negatively charged dangling bond. Similar to the process above a single common cut in $\Delta f$ was used to assign charge states to the normalized data (step 6).

Height-dependent contrast in $\Delta \boldsymbol{f}$ images: To provide additional insight to the qualitative behaviour of the $\Delta f$ signal above each species we measured site-specific force curves. The results are presented as Figure $3 \mathrm{a}$ and in Supporting Figure $\mathrm{S} 5$ which shows $\Delta f(z)$ signals obtained while approaching the tip to the corresponding sites. For large tip-surface separations (height range $>0 \mathrm{pm}$ ) all three species exhibit an identical frequency shift, i.e. the $\Delta f(z)$ curves coincide and there is no contrast. As the tip approaches (approx. -200 to $0 \mathrm{pm}$ ), attractive van der Waals forces are observed first on the hydrogen atoms. As a result, vacancies and neutral DBs have smaller $|\Delta f|$ compared to the surrounding $\mathrm{H}$ atoms and appear as bright protrusions in $\Delta f$ images taken in this height range. Negatively charged DBs appear dark (large $|\Delta f|$ ) due to electrostatic contributions.

For tip offsets closer to the surface than $-200 \mathrm{pm}$, the tip enters the repulsive regime over hydrogen atoms. Consequently, the $|\Delta f|$ obtained over the neutral dangling bond becomes greater than that of the hydrogen resulting in a darker appearance of the DBs. The final feature of interest is a sharp step (approx. $-300 \mathrm{pm}$ ) in the approach curve on the neutral DB, which we attribute to the charging of the dangling bond beneath the tip and the dark appearance (large $|\Delta f|$ ). As the tip is being retracted the charge remains at the site under the tip and the corresponding DB appears darker than hydrogen or neutral DBs in $\Delta f$ images. All images and line profiles for the write and read scans are well within the same height regime and no contrast inversion exists.

Frequency to force conversion: To reliably separate the short range forces measured over dangling bonds and hydrogen we took reference measurements over dimer vacancies (Fig. S4). [2, 3] We 
performed our measurements of $\Delta f(z)$ at the various sites in a series while keeping the instrument in constant height mode. Repeatedly taking $\Delta f(z)$ spectra over hydrogen atoms throughout the experiment allowed us to determine the residual thermal drift and piezo artefacts. Afterwards we shifted the spectra relative to one another and calculated the minimum residual sum squared error of the shifted spectra

$$
\Sigma\left(\Delta f(z)_{\text {shifted }}-\Delta f(z)_{\text {reference }}\right)^{2} \text {. }
$$

By comparing the determined optimum offset for each hydrogen spectrum we revealed linear drift within each set of measurements, which subsequently allows us to correct the tip offsets.

We used the Sader-Jarvis method [4] and corrections proposed by Giessibl [5] to convert the $\Delta f(z)$ spectra to force curves (Fig. S5). We assumed a cantilever stiffness of $1800 \mathrm{~N} / \mathrm{m}$ but note that others have reported large uncertainties in this value. The uncertainty in our reported short range forces correspond to the standard deviation between the data and their optimal fit and so a linear correction to the forces and their corresponding uncertainties would result if a different stiffness was assumed.
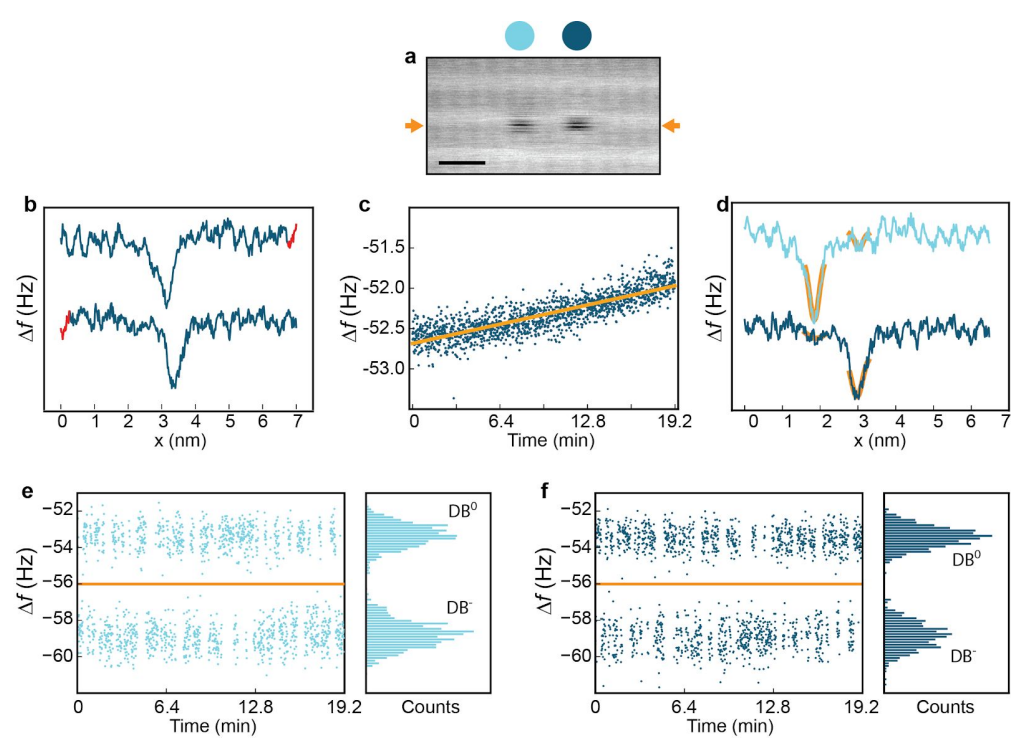

Figure S1: Illustration of data processing routine for $\Delta \boldsymbol{f}$ maps. (a) Constant height $\Delta f$ image of a dangling bond structure taken at $0 \mathrm{~V}$. The initial tip height is set on a hydrogen atom at $-1.8 \mathrm{~V}$ and $50 \mathrm{pA}$ before moving the tip 300 $\mathrm{pm}$ towards the surface. The scale bar is $1 \mathrm{~nm}$. (b) Two sequential $\Delta f$ line scans demonstrate negative charge confined to the right-hand dangling bond (line scan width is larger than the window shown in (a), line scans are offset for clarity). The peaks are not aligned because they correspond to forward (top) and backward (bottom) line scans, which typically have a fixed offset due to piezo creep. The red tails on both line scans demonstrate the data that is chopped to align the scans. (c) The average $\Delta f$ of each line scan over the course of the entire experiment demonstrates that the tip was slowly drifting away from the sample. A linear fit of this data (orange line) is subtracted from the dataset. (d) Each line scan is fit with two gaussian peaks to extract the $\Delta f$ over each dangling bond (colour legend indicated above (a)). Note that for neutral dangling bonds this corresponds to a fit of the signal associated with hydrogen/noise. (e-f) The $\Delta f$ extracted for each dangling bond clearly displays two distinct states, which we assign to the negative and neutral charge states of each dangling bond. Each histogram has 75 equal width bins between $\Delta f=-62$ and $-51 \mathrm{~Hz}$, and has an integrated area of 1.0 . 

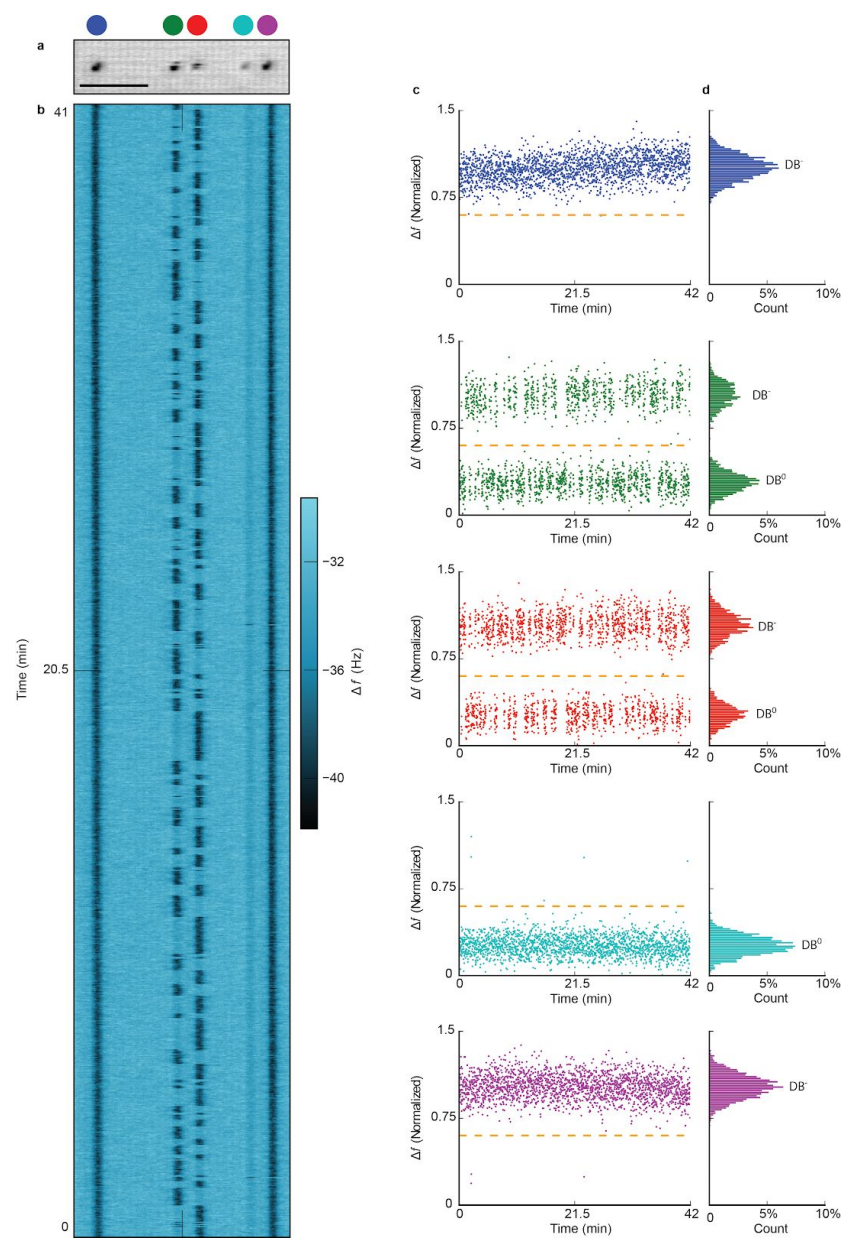

Figure S2: Digitization of line scans of an asymmetric structure composed of five dangling bonds. (a) Constant height $\Delta f$ image of the dangling bond structure at $0 \mathrm{~V}$. (b) A line scan map composed of $2048 \Delta f$ line scans acquired in the read-regime $(-300 \mathrm{pm})$ over structure demonstrate. (c) The normalized $\Delta f$ acquired over each dangling bond throughout the course of the experiment demonstrates clearly that there are two charge states of each dangling bond (although only the green and red dangling bonds appear to fluctuate between them). With the normalizing procedure described above the negative dangling bond charge state is normalized to a $\Delta f$ of 1.0 , and the $\Delta f$ of the neutral dangling bond state is centered approximately at 0.25 . The orange dotted lines demonstrate that a single common cut of $\Delta f=0.6$ in the normalized data can be used to digitize the charge state of the structure with each line scan. (d) Histograms of the normalized $\Delta f$ for each dangling bond reveal that the $\Delta f$ corresponding to the two charge states of each dangling bond have a Gaussian distribution. Upon assigning binary numbers to this dataset it was found that in $<1 \%$ of the line scans the charge configuration corresponded to having a third negative charge in the four paired dangling bonds. Each histogram has 75 equal width bins between $\Delta f$ (normalized) $=0$ and 1.5 , and has an integrated area of 1.0 . 
b

a
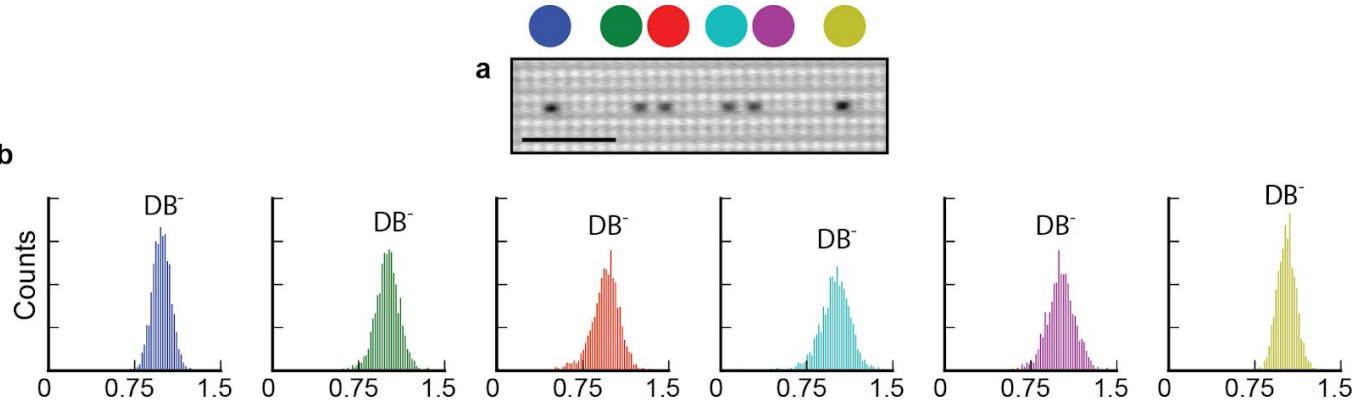

C
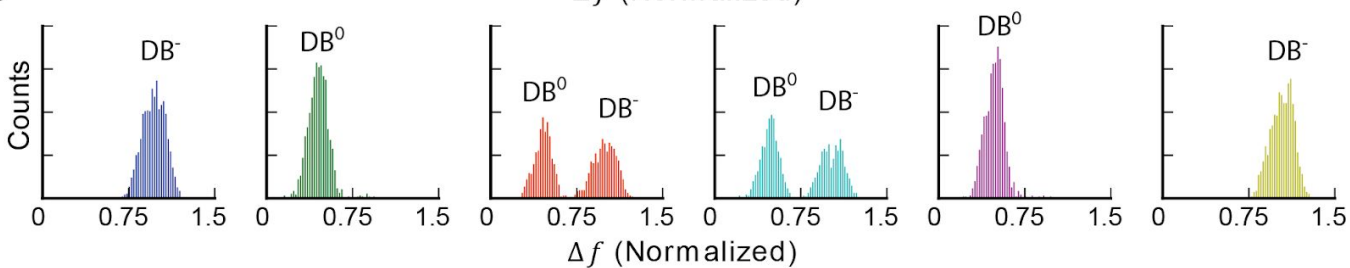

Figure S3: Histograms of the normalized $\Delta f$ measured over each site in a symmetric six dangling bond structure at different tip heights. (a) Constant height $\Delta f$ image of the structure. (b, c)Histograms of the normalized $\Delta f$ measured over each dangling bond at (b) $\mathrm{z}=-320 \mathrm{pm}$ and (c) $\mathrm{z}=-270 \mathrm{pm} .1600$ line scans at both heights were used to gather statistics. Each histogram has 75 equal width bins between $\Delta f$ (normalized) $=0$ and 1.5 , and has an integrated area of 1.0. All the dangling bonds appear negatively charged in (b). In (c), the isolated dangling bonds on either end (blue and yellow) remain negatively charged while the outer atoms of each pair (green and purple) are neutral. In (c) the inner atoms (red and cyan) fluctuate between the neutral and negative charge states; the integrated area of each peak is approximately 0.5 , indicating they are equally likely to be in the neutral or negative charge state. This can be seen directly in Fig. $2 \mathrm{~d}$ where a single electron switches between these two dangling bonds.

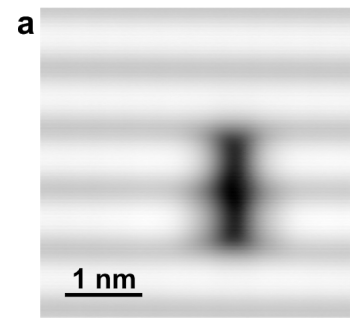

$157 \mathrm{pm} \rightleftharpoons 335 \mathrm{pm}$
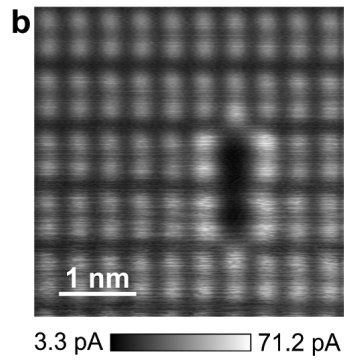

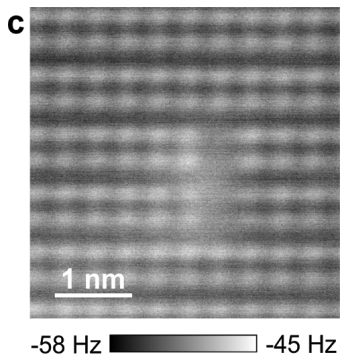

$-58 \mathrm{~Hz} \longrightarrow-45 \mathrm{~Hz}$

Figure S4: Dimer vacancy in neighboring dimer rows on hydrogen-terminated $\mathrm{Si}(100)$. (a) Constant current filled state STM image, $-1.8 \mathrm{~V}$ and $50 \mathrm{pA}$. (b) Constant height tunneling current image ( $300 \mathrm{mV}$, tip offset $-370 \mathrm{pm})$. (c) Constant height $\Delta f$ image, $0 \mathrm{~V}$ and tip offset $-300 \mathrm{pm}$. Reference tip height for (b) and (c) is $-1.8 \mathrm{~V}$ and $50 \mathrm{pA}$ measured above hydrogen. 
a
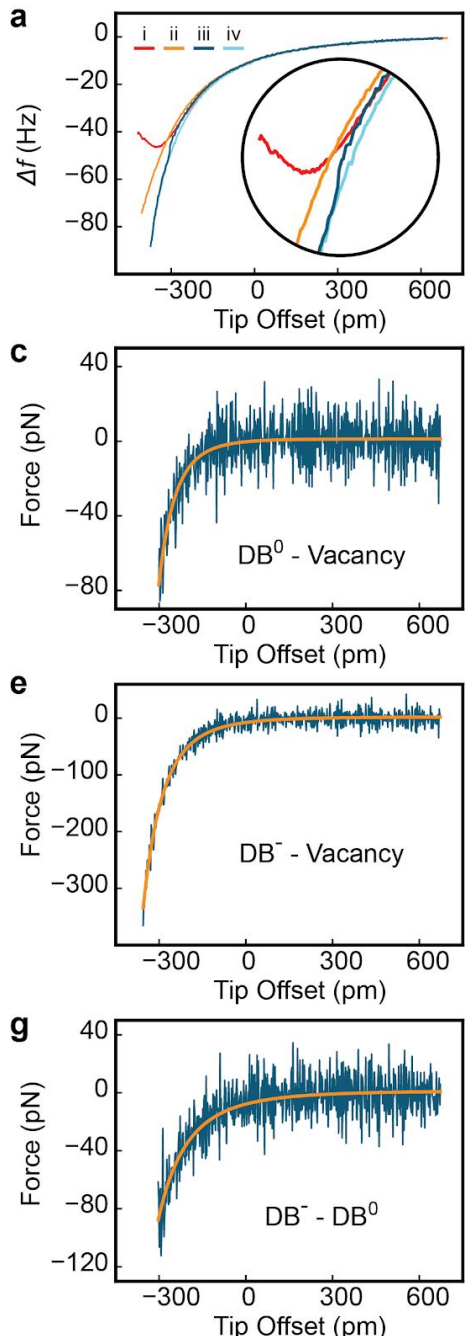
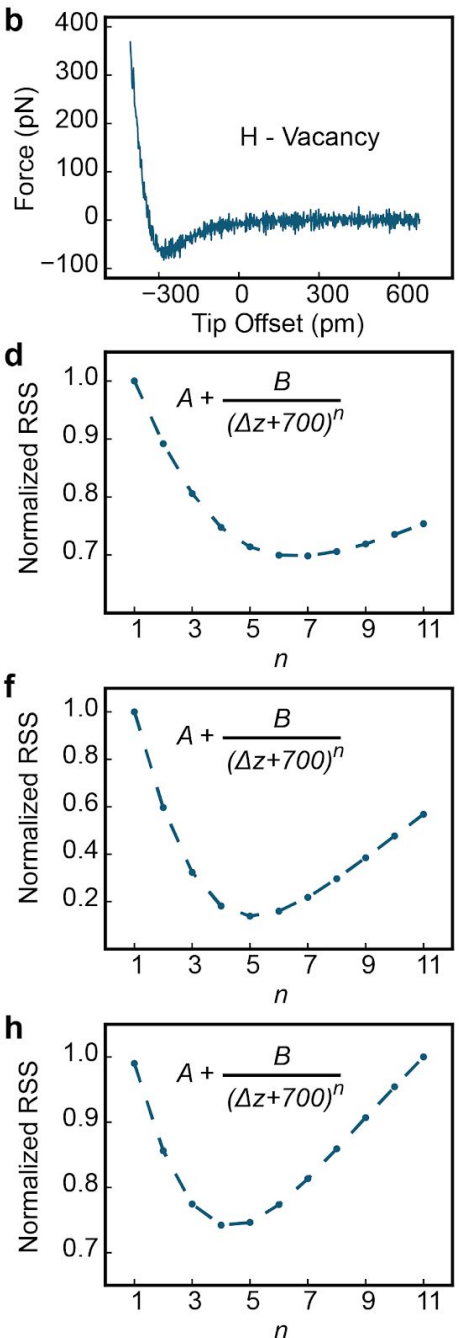

Figure S5: Extraction of short range forces from $\Delta f(z)$ spectra. (a) $\Delta f(z)$ taken over a hydrogen atom (i. red), dimer vacancy (ii. orange) and the right dangling bond of a pair (iii. dark blue, approach; iv. light blue, retract). $\Delta z=$ 0 corresponds to an STM setpoint of- $1.8 \mathrm{~V}$ and $50 \mathrm{pA}$. All curves were taken at $0 \mathrm{~V}$. Inset: a closeup of the spectra near $\Delta z=-300 \mathrm{pm}$. (b) The short range force measured over a hydrogen atom, corresponding to the difference in the force measured over the hydrogen atom and the dimer vacancy (forces found by converting $\Delta f(z)$ to force via Sader-Jarvis Method [4,5]. (c, e, g) The short range forces measured over a neutral and negatively charged dangling bond. The force for the neutral dangling bond corresponds to the approach curve in (a) (dark blue) up to the point of the sudden increase in $|\Delta f|$. The force for the negatively charged dangling bond corresponds to the retract curve in (a) (light blue). (d, f, h) Comparisons of the fits of the extracted short range forces for (c, e, g), respectively. RSS corresponds to the residual sum of squares error. The absolute tip height at the set point was determined to be 700 $\mathrm{pm}$. Approaching the tip $700 \mathrm{pm}$ from our setpoint typically results in sudden increases to the tunneling current and small changes to the tip apex and surface, strongly suggesting the tip makes direct contact with the hydrogen-free silicon surface. 

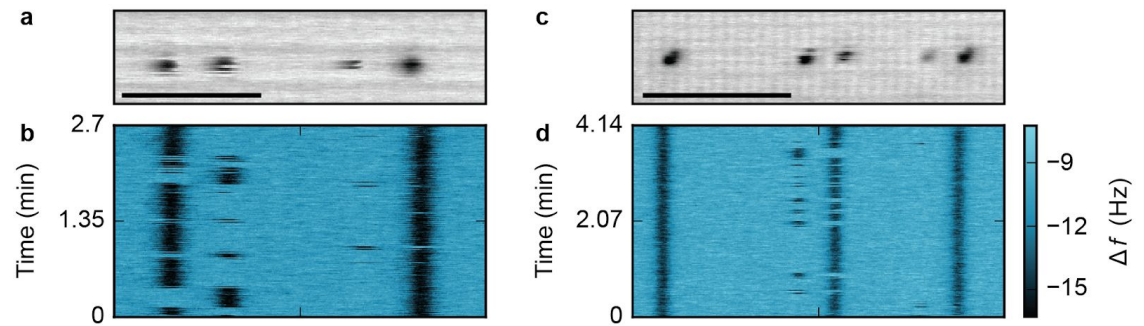

Figure S6: The influence of adding an isolated dangling bond on the polarization of dangling bond pairs. (a) Constant height $\Delta f$ image of a symmetric structure composed of four dangling bonds. (b) A line scan map composed of two hundred sequential $\Delta f$ line scans acquired over the structure demonstrate that it is naturally polarized. The negative charge confined to the left-hand pair favors the outer dangling bond but occasionally fluctuates to the inner dangling bond. The negative charge confined to the right-hand pair almost exclusively occupies the outer dangling bond. (c) An isolated dangling bond was added to the left of the same structure in (a) using STM lithography. (d) A line scan map composed of two hundred sequential $\Delta f$ line scans acquired over the structure demonstrate the effect of this additional negative charge to the polarization of the structure. The right-hand pair remains polarized in the same way as (b). The polarization of the left-hand pair reverses compared to (b). This is easily rationalized by noting that the new dangling bond acts as a local Coulombic bias. This demonstrates that local charges (e.g. negatively charged dangling bonds or ionized donors) can influence the distribution of charge configurations these structures display. The scale bars in (a) and (c) are 3 and $4 \mathrm{~nm}$, respectively. The individual line scans acquired in (d) are longer than in (b) due to the increased distance the tip has to move. The $\Delta f$ colour bar applies to both (b) and (d).

\section{References}

[1] T. R. Huff, H. Labidi, M. Rashidi, M. Koleini, R. Achal, M. H. Salomons, and R. A. Wolkow, ACS Nano 11, 8636 (2017).

[2] S. R. Schofield, N. J. Curson, J. L. O’Brien, M. Y. Simmons, R. G. Clark, N. A. Marks, H. F. Wilson, G. W. Brown and M. E. Hawley, Phys. Rev. B 69, 085312 (2004).

[3] N. J. Curson, S. R. Schofield, M. Y. Simmons, L. Oberbeck, J. L. O’Brien, and R. G. Clark, Phys. Rev. B 69, 195303 (2004).

[4] J. E. Sader and S. P. Jarvis, Appl. Phys. Lett. 84, 1801-1803 (2004).

[5] J. Welker, E. Illek and F. J. Giessibl, Beilstein J. Nanotechnol. 3, 238-248 (2012). 